\title{
ELASTIC DEFORMATION OF POLYCRYSTALS
}

\author{
Rajeev Ahluwalia, ${ }^{1}$ Turab Lookman, ${ }^{1}$ and Avadh Saxena ${ }^{1}$ \\ ${ }^{1}$ Theoretical Division, Los Alamos National Laboratory, Los Alamos, New Mexico, 87545
}

(Dated: October 31, 2018)

\begin{abstract}
We propose a framework to model elastic properties of polycrystals by coupling crystal orientational degrees of freedom with elastic strains. Our model encodes crystal symmetries and takes into account explicitly the strain compatibility induced long-range interaction between grains. The coupling of crystal orientation and elastic interactions allows for the rotation of individual grains by an external load. We apply the model to simulate uniaxial tensile loading of a $2 D$ polycrystal within linear elasticity and a system with elastic anharmonicities that describe structural phase transformations. We investigate the constitutive response of the polycrystal and compare it to that of single crystals with crystallographic orientations that form the polycrystal.
\end{abstract}

PACS numbers:

A study of the mechanical properties of polycrystals is important as most technologically important materials exist in a polycrystalline state. A polycrystal is an aggregate of grains that have different crystallographic orientations. The properties of a polycrystal depend on its texture (distribution of crystallographic orientations). It is desirable to understand how the strains are distributed due to an applied external load and how this influences the average elastic moduli of the polycrystalt. An important feature of some polycrystalline metallic alloys and ceramics is the presence of domain walls within the grains due to an underlying martensitic transition. This microstructure influences the response of the material to an external load. For example, in martensites, the deformation is accompanied by the motion of the domain walls. Texture evolution caused by rotation of grains under the application of an external load is another important factor that influences the mechanical behavior of a polycrystal. Polycrystalline specimens exhibit significant grain rotation in the plastic regime to accommodate crystallographic slip. Grain rotations H $^{2}$ to $\sim 1^{\circ}$ have also been observed in atomistic simulations nanocrystals, even at low strains of $\sim 4 \%$.

The problem of finding the effective properties of polycrystals has been studied by analytical methodsw. However, the complex geometry of a polycrystal or the long-range elastic interactions between the grains are often not accounted for in these methods. In fact, these approaches are only able to give bounds on the effective properties. Recently, the mechanical deformation of polycrystals has been studied by atomistic simulation $\$$ which have been limited to nano-sized grains. Simulating bulk systems with atomistic simulations requires enormous computational power and hence continuum simulations that can cover a range of intermediate length scales are essential for describing the microstructure at the sub-micron scale. Several phase-field models have been proposed to model grain growth phenomena 6 - 8 . Although these models correctly describe the grain morphologies and the domain growth laws, the issues of elasticity and material specific crystal symmetries are usually not addressed. Recently Elder et al. 1 studied elastic and plastic effects using a model formulated to describe pattern selection. The model, in terms of particle density fields, is specific only to certain symmetries that are selected by appropriate choice of wavelengths in the free energy, which does not contain experimentally measured quantities such as elastic constants. In this Letter, we propose a polycrystal model based on continuum elasticity that can be applied to any crystal symmetry and has the appropriate single-crystal elastic constants as input parameters. In this model, elastic strains are coupled to a phase field model through an orientation field that is determined from a multi-component order parameter describing the crystal orientations. Due to this coupling, the strains in each grain as well as the grain orientations can change under an external load. This experimentally relevant feature is not accounted for in models that consider static grains created by Voronoi construction 10 . In the present work, we determine the mechanical properties of linear elastic materials and those described by nonlinear elasticity, such as martensites.

The free-energy functional is written as $F=F_{\text {grain }}+$ $F_{\text {elastic }}+F_{\text {load }}$, where $F_{\text {grain }}$ is the free energy density due to the orientational degrees of freedom of the polycrystal, $F_{\text {elastic }}$ represents the elastic free energy and $F_{\text {load }}$ is the free energy contribution due to an external applied load. The polycrystalline system is described by a set of $Q$ non-conserved order parameters $\left(\eta_{1}, \eta_{2}, \ldots, \eta_{Q}\right)$. A given grain orientation corresponds to one of the $Q$ order parameters being positive nonzero while the rest are zero. The free energy $F_{\text {grain }}$ is given by

$$
\begin{aligned}
F_{\text {grain }} & =\int d \vec{r}\left\{\sum_{i=1}^{Q}\left[\frac{a_{1}}{2} \eta_{i}{ }^{2}+\frac{a_{2}}{3} \eta_{i}{ }^{3}+\frac{a_{3}}{4} \eta_{i}{ }^{4}\right]\right. \\
& \left.+\frac{a_{4}}{2} \sum_{i=1}^{Q} \sum_{j>i}^{Q} \eta_{i}{ }^{2} \eta_{j}{ }^{2}+\sum_{i=1}^{Q} \frac{K}{2}\left(\nabla \eta_{i}\right)^{2}\right\} .
\end{aligned}
$$

For $a_{1}, a_{2}<0$ and $a_{3}, a_{4}>0$, the first two terms in equation (1) describe a potential with $Q$ degenerate minima corresponding to $Q$ grain orientations. The gradient energy $(K>$ $0)$ represents the energy cost of creating a grain boundary. It is also possible to associate an orientational field $\theta(\vec{\eta}, \vec{r})$, where

$$
\theta(\vec{\eta}, \vec{r})=\frac{\theta_{m}}{Q-1}\left[\frac{\sum_{i=1}^{Q} i \eta_{i}}{\sum_{i=1}^{Q} \eta_{i}}-1\right] .
$$

Thus, there are $Q$ orientations between 0 and $\theta_{m}$. For the elastic free energy the linearized strain tensor in a global 
reference frame is defined by $\epsilon_{i j}=\left(u_{i, j}+u_{j, i}\right) / 2(i=$ 1,2: $j=1,2)$, where $u_{i}$ represents $i$ th component of the displacement vector and $u_{i, j}$ is its $j$ th displacement gradient. For illustration, we consider a $2 D$ lattice with square symmetry and use the symmetry-adapted linear combinations of the strain tensor defined as $\epsilon_{1}=\left(\epsilon_{x x}+\epsilon_{y y}\right) / \sqrt{2}$, $\epsilon_{2}=\left(\epsilon_{x x}-\epsilon_{y y}\right) / \sqrt{2}$ and $\epsilon_{3}=\epsilon_{x y}$. To generalize this theory for the case of a polycrystal, the strain tensor in a rotated frame is calculated as $R(\theta(\vec{\eta})) \in R^{T}(\theta(\vec{\eta}))$, where $R(\theta(\vec{\eta}))$ is a rotation matrix. Using this transformation, the elastic free energy in a global frame of reference is $F_{\text {elastic }}=\int d \vec{r}\left\{\frac{A_{1}}{2} e_{1}^{2}+\frac{A_{2}}{2} e_{2}^{2}+\frac{A_{3}}{2} e_{3}^{2}+f_{n l}\left(e_{1}, e_{2}, e_{3}\right)+\right.$ $\left.\frac{K_{2}}{2}\left(\nabla e_{2}\right)^{2}+\frac{K_{3}}{2}\left(\nabla e_{3}\right)^{2}\right\}$ where $e_{1}, e_{2}, e_{3}$ are defined as $e_{1}=\epsilon_{1}, e_{2}=\epsilon_{2} \cos [2 \theta(\vec{\eta})]+\sqrt{2} \epsilon_{3} \sin [2 \theta(\vec{\eta})]$ and $e_{3}=$ $-(1 / \sqrt{2}) \epsilon_{2} \sin [2 \theta(\vec{\eta})]+\epsilon_{3} \cos [2 \theta(\vec{\eta})]$. The orientation field $\theta(\vec{\eta})$ is determined from the minima of free energy in (1) using (2). Here $A_{1}=C_{11}+C_{12}, A_{2}=C_{11}-C_{12}$ and $A_{3}=4 C_{44}$, where $C_{11}, C_{12}$ and $C_{44}$ are the elastic constants for a crystal with square symmetry. $K_{2}$ and $K_{3}$ are the appropriate gradient coefficients that in principle can be obtained from experimentally measured phonon dispersion data. The term $f_{n l}\left(e_{1}, e_{2}, e_{3}\right)$ represents the nonlinear part of the elastic free energy and is crucial in describing structural phase transitions.

In this work, we are interested in simulating a uniaxial loading experiment. If we choose the $x$ axis to be the loading direction, the free energy contribution due to the external load is $F_{\text {load }}=-\int d \vec{r} \sigma \epsilon_{x x}=-\int d \vec{r} \frac{\sigma}{\sqrt{2}}\left(\epsilon_{1}+\epsilon_{2}\right)=-\int d \vec{r} \frac{\sigma}{\sqrt{2}}\left(e_{1}+\right.$ $\left.e_{2} \cos [2 \theta(\vec{\eta})]-\sqrt{2} e_{3} \sin [2 \theta(\vec{\eta})]\right)$. The strains $\epsilon_{1}, \epsilon_{2}$ and $\epsilon_{p}$ are not independent but satisfy a compatibility relationship 2 : $\nabla^{2} \epsilon_{1}-\left(\frac{\partial^{2}}{\partial x^{2}}-\frac{\partial^{2}}{\partial y^{2}}\right) \epsilon_{2}-\sqrt{8} \frac{\partial^{2}}{\partial x \partial y} \epsilon_{3}=0$. Using a method introduced earlier for single crystal martensitic transformation 13 , the strain $e_{1}$ may be eliminated using compatibility, to express the effective free energy $F_{\text {eff }}=F_{\text {elastic }}+F_{\text {load }}$ as

$$
\begin{aligned}
F_{\text {eff }} & =\frac{A_{1}}{2} \int d \vec{k}\left[C_{2}^{2}(\vec{k})\left|\Gamma_{2}(\vec{k})\right|^{2}+C_{3}^{2}(\vec{k})\left|\Gamma_{3}(\vec{k})\right|^{2}\right. \\
& \left.+C_{2}(\vec{k}) C_{3}(\vec{k})\left[\Gamma_{3}(\vec{k}) \Gamma_{2}(-\vec{k})+\Gamma_{3}(-\vec{k}) \Gamma_{2}(\vec{k})\right]\right] \\
& +\int d \vec{r}\left[\frac{A_{2}}{2} e_{2}{ }^{2}+\frac{A_{3}}{2} e_{3}^{2}+f_{n l}\left(e_{2}, e_{3}\right)\right. \\
& +\frac{K_{2}}{2}\left(\nabla e_{2}\right)^{2}+\frac{K_{3}}{2}\left(\nabla e_{3}\right)^{2} \\
& \left.-\frac{\sigma}{\sqrt{2}}\left(e_{2} \cos [2 \theta(\vec{\eta})]-\sqrt{2} e_{3} \sin [2 \theta(\vec{\eta})]\right)\right] .
\end{aligned}
$$

where $\Gamma_{2}(\vec{k}), \quad \Gamma_{3}(\vec{k})$ represent Fourier transforms of $e_{2} \cos [2 \theta(\vec{\eta})]-\sqrt{2} e_{3} \sin [2 \theta(\vec{\eta})]$ and $e_{2}(\sin [2 \theta(\vec{\eta})] / \sqrt{2})+$ $e_{3} \cos [2 \theta(\vec{\eta})]$ respectively, $C_{2}(\vec{k})=\left(k_{x}{ }^{2}-k_{y}{ }^{2}\right) /\left(k_{x}{ }^{2}+k_{y}{ }^{2}\right)$ and $C_{3}(\vec{k})=\sqrt{8} k_{x} k_{y} /\left(k_{x}{ }^{2}+k_{y}{ }^{2}\right)$. The long-range terms ensure that compatibility is satisfied within the grains as well as at the grain boundaries.

The dynamics of the grains is given by $Q$ equations

$$
\frac{\partial \eta_{i}}{\partial t}=-\gamma_{\eta} \frac{\delta F}{\delta \eta_{i}}
$$

where $\gamma_{\eta}$ is a dissipation coefficient and $i=1, \ldots, Q$ correspond to $Q$ grain orientations. The corresponding overdamped dynamics for the strains is

$$
\frac{\partial e_{2}}{\partial t}=-\gamma_{2}\left[\frac{\delta F}{\delta e_{2}}\right], \frac{\partial e_{3}}{\partial t}=-\gamma_{3}\left[\frac{\delta F}{\delta e_{3}}\right],
$$

where $\gamma_{2}$ and $\gamma_{3}$ are the appropriate dissipation coefficients for the strains and $F=F_{\text {grain }}+F_{\text {eff }}$ is the total free energy of the system.

The mechanical properties of many materials are well described by the harmonic approximation for which the nonlinear term $f_{n l}\left(e_{1}, e_{2}, e_{3}\right)=0$. For a homogeneous single crystal $e_{2}, e_{3}$ and $\vec{\eta}$ are constant and for $\mathrm{Cu} A_{1}=289.8$ GPa, $A_{2}=47.0 \mathrm{GPa}$ and $A_{3}=301.6 \mathrm{GPa}\left(C_{11}=168.4\right.$ $\left.\mathrm{GPa}, C_{12}=121.4 \mathrm{GPa}, C_{44}=75.4 \mathrm{GPa}\right)$. For the parameters in $F_{\text {grain }}$ we choose $a_{1}=a_{2}=-A_{2}, a_{3}=A_{2}$, $a_{4}=2 A_{2}, Q=5$ and $\theta_{m}=45^{\circ}$. We choose the gradient coefficients in terms of an arbitrary length scale $\delta$ so that $K=K_{2}=K_{3}=A_{2} \delta^{2}$ and lengths are scaled by $\vec{r}=\delta \vec{\zeta}$. The free energy in (1) then has five degenerate minima defined by $\theta_{0}(\vec{\eta})=0^{\circ}, 11.25^{\circ}, 22.5^{\circ}, 33.75^{\circ}, 45^{\circ}$, corresponding to five different grain orientations.

To study the polycrystal, we first generate an initial polycrystalline configuration by solving (4) and (5) with $\sigma=0$, using random initial conditions. For all simulations in this paper, we assume $\gamma_{\eta}=\gamma_{1}=\gamma_{2}=\gamma$ and use rescaled time $t^{*}=t\left|A_{2}\right| \gamma$. For $\sigma=0$, the elastic effects do not influence the grain growth as all the strains vanish. Grains with orientations $\theta_{0}(\vec{\eta})=0^{\circ}, 11.25^{\circ}, 22.5^{\circ}, 33.75^{\circ}, 45^{\circ}$ nucleate and coarsen. We arrest the system in a given configuration by suddenly changing the value of the parameter $a_{1}$ from $-A_{2}$ to $-16 A_{2}$. This increases the free-energy barriers between the crystalline states and the growth stops. With the arrested polycrystal configuration as the initial condition, we simulate a quasi-static uniaxial tensile loading using (4) and (5). The stress $\sigma$ is varied in steps of $0.06 \mathrm{GPa}$ and we let the strains relax after each change for $t^{*}=25$ steps. Figure 1(a) shows the spatial distribution of the polycrystal orientation $\theta_{\sigma}(\vec{r})$ at a loading of $\sigma=2.35 \mathrm{GPa}$ for a system of size $128 \delta \times 128 \delta$. We note that there is no significant motion of the grain boundaries from the initial arrested configuration to the configuration depicted in Fig. 1(a) (the individual grains have rotated by a small amount $\left(\sim 0.01^{\circ}\right)$, consistent with the coupling between stress and the orientation). In Fig. 1(b), we show the corresponding distribution of uniaxial strain $\epsilon_{x x}$. The strain distribution is anisotropic as $\epsilon_{x x}$ in a grain depends on the orientation.

In Fig. 2, we show the variation of the average strain $\left\langle\epsilon_{x x}\right\rangle$ with the load $\sigma$. For comparison, we also plot the analogous single crystal curves with crystallographic orientations that constitute the polycrystal configuration in Fig. 1 (single crystal simulations were performed using only one orientation but with identical free energy parameters and loading rate as the polycrystal). The Young's modulus of the simulated polycrystal was $\sim 126 \mathrm{GPa}$. This is in the range of experimentally measured values of $124 \mathrm{GPa}{ }^{14}$ and $129.8 \mathrm{GPall}$ quoted for bulk polycrystalline $\mathrm{Cu}$. The result is not sensitive to the 
choice of parameters for the polycrystal phase field model, at least in the linear elastic regime.

Another important class of materials that can be studied using this approach are martensites that undergo a displacive structural phase transformation. The transformed phase is characterized by a complex arrangement of crystallographic variants known as twins. We consider the case of a $2 D$ square to rectangle transition for which the deviatoric strain $e_{2}$ is the appropriate order parameter. For the high temperature square phase $e_{2}=0$ and for the low temperature martensitic phase $e_{2}= \pm e_{0}$, corresponding to the two rectangular variants. This system exhibits the so called shape-memory effect which is governed by the motion of martensitic domains. The microstructure depends on the underlying crystal symmetry and hence the displacements and domain wall orientations of the atoms in each grain depend on the grain orientation. Thus the shape memory effect will be influenced by the texture and hence it is important to compare the mechanical response of single and polycrystal martensites.

The anharmonic contribution to the elastic free energy is given by $f_{n l}=\frac{\alpha}{4} e_{2}{ }^{4}+\frac{\beta}{6} e_{2}{ }^{6}$ and describes a first order transition for $\alpha<0$. We choose $A_{1}=140 \mathrm{GPa}, A_{3}=280$ GPa, $\alpha=-1.7 \times 10^{4} \mathrm{GPa}$ and $\beta=3 \times 10^{7} \mathrm{GPa}$, parameters that correspond to $\mathrm{FePd} 16$. The constant $A_{2}$ depends on the temperature and we choose $A_{2}=-3 \mathrm{GPa}$, a temperature in the martensitic phase. The measured gradient coefficient $K_{2} / a_{0}{ }^{2}=25 \mathrm{GPa}$, where $a_{0}$ is the lattice spacing of the crystal and assume $K_{3}=0$ since the deviatoric strain is the dominant mode of deformation. The parameters for $F_{\text {grain }}$ are $a_{1}=a_{2}=-\left|A_{2}\right|, a_{3}=\left|A_{2}\right|, a_{4}=2\left|A_{2}\right|$ and the grain boundary coefficient is chosen in terms of the lattice spacing $a_{0}$ to be $K / a_{0}^{2}=10^{4}\left|A_{2}\right|$. The space variable is rescaled by introducing a dimensionless length scale $\vec{\zeta}$ so that $\vec{r}=\left(100 a_{0}\right) \vec{\zeta}$. The maximum orientation is chosen to be $\theta_{m}=30^{\circ}$ so for $Q=5$, the allowed orientations are $0^{\circ}$, $7.5^{\circ}, 15^{\circ}, 22.5^{\circ}$ and $30^{\circ}$. Employing the above set of parameters, we simulate the martensitic domain structures using (4) and (5). With same procedure as for the linear elastic case, a stable polycrystal configuration is obtained. When the applied stress $\sigma=0$, the parameter $A_{2}=-3 \mathrm{GPa}$ ensures that the system is well in the martensite phase and domains of the two rectangular variants (twins) are formed. After obtaining a stable martensitic polycrystal, the loading process is simulated by quasi-statically varying the stress in steps of $0.38 \mathrm{GPa}$ upto a maximum stress $15 \mathrm{GPa}$ (after each stress change, the system is allowed to relax for $t^{*}=25$ steps). The system is then unloaded by decreasing the stress to zero at the same rate.

Figure 3 shows the evolution of variants and the grains at different stress levels during the loading-unloading process for a system of size $12800 a_{0} \times 12800 a_{0}$. The left column shows the distribution of $\epsilon_{2}(\vec{r})$ (deviatoric strain in a global frame) and the right column shows the corresponding distribution of the orientations $\theta(\vec{r})$. It is clear from Fig. 3 that the domain wall orientations depend on the orientation of a grain. On loading, the simulated polycrystal starts to detwin (favored variants grow at the expense of unfavored ones). However, even at the maximum load of $\sigma=15 \mathrm{GPa}$, some unfavorable variants persist. On complete unloading, a domain structure is nucleated again due to inhomogeneities in the polycrystal. However, this domain structure is not the same as that before the loading indicating an underlying hysteresis. The orientation distribution is also influenced by the external load, as can be seen in the right column of Fig. 3. The grains with large misorientation with the loading axis rotate significantly $\left(\sim 10^{\circ}\right)$ while the grains having lower orientation do not rotate as much. The mechanism of this rotation is the desire of the system to maximize strain in the loading direction so as to minimize the elastic free energy. At high stress, some grain boundaries start moving to accommodate the applied stress, as is clear from the orientation distribution at $\sigma=15 \mathrm{GPa}$.

The stress-strain curve corresponding to Fig. 3 is shown in Fig. 4. Also shown are single crystal curves for all five orientations that constitute the polycrystal of Fig. 3. We observe that the hysteresis for the polycrystal case is much smaller than that for a single crystal oriented along the loading axis. Our findings regarding the hysteresis are consistent with the fact that polycrystals haye poor shape memory properties compared to single crystals5. The simulations also indicate that grain rotations will influence the mechanical properties of shape memory alloys. Recently, in situ measurements of texture evolution during compression experiments on $\mathrm{Ni}-\mathrm{Ti}$ shape memory alloys 17 have been reported. However, these experiments cannot predict whether the changes in texture are due to detwinning or rotation of grains. Our simulations show that both these processes can contribute to texture evolution.

In summary, we have proposed a framework to study the mechanical properties of polycrystals in which the long-range elastic interaction between grains and the connectivity of the microstructure is taken into account. The approach can be extended to any crystal symmetry or loading (e.g., shear) and does not require any a priori assumption of grain shapes or the microstructure. An important feature of our work is the coupling between the grain orientation and elasticity. We have applied the model to study mechanical properties of linear elastic and martensitic materials. For the linear elastic case the observed grain rotations are small $\left(\sim 0.01^{\circ}\right)$ and hence do not influence the mechanical properties. In contrast, the martensitic case shows significant grain rotation $\left(\sim 10^{\circ}\right)$ due to accommodation of the transformation strain. This behavior is sensitive to the choice of parameters of the polycrystal model (energy barriers between grains) and therefore determination of these parameters from experiment or atomistic simulations will allow accurate prediction of mechanical properties.

We thank Kai Kadau and R.C. Albers for discussions. This work was supported by the U.S. Dapartment of Energy.
${ }^{1}$ M Avellaneda, A. V. Cherkaev, L. V. Gibiansky, G. W. Milton,
and M. Rudelson, J. Mech. Phys. Solids 44, 1179 (1996).
${ }^{2}$ L. Margulies, G. Winther, and H. F. Poulsen, Science 291, 2392 
(2001).

3 J. Schiotz, T. Vegge, F. D. Di Tolla, and K. W. Jacobsen, Nature (London) 391, 561 (1998); Phys. Rev. B 60, 11971 (1998).

${ }^{4}$ H. V. Swygenhoven, Science 296, 67 (2002).

5 K. Bhattacharya and R. V. Kohn, Arch. Rational Mech. Anal. 139, 99 (1997); Y. C. Shu and K. Bhattacharya, Acta Mater. 46, 5457 (1998).

${ }^{6}$ L. Q. Chen and W. Wang, Phys. Rev. B 50, 15752 (1994).

7 B. Morin, K. R. Elder, M Sutton, and M. Grant, Phys. Rev. Lett. 75, 2156 (1995).

8 J. A. Warren, W. C. Carter, and R. Kobayashi, Physica (Amsterdam) 261A, 159 (1998).

9 K. R. Elder, M. Katakowski, M. Haataja, and M. Grant, Phys. Rev. Lett. 88, 245701 (2002).

${ }^{10}$ Y. M. Jin, A. Artemev, and A. G. Khachaturyan, Acta Mater. 49, 2309 (2001); A. Artemev, Y. M. Jin, and A. G. Khachaturyan, Philos. Mag. A 82, 1249 (2002).

${ }^{11}$ G. R. Barsch and J. A. Krumhansl, Phys. Rev. Lett. 53, 1069 (1984).

12 E. A. H. Love, A Treatise on the Mathematical Theory of Elasticity (Dover, New York, 1944).

13 T. Lookman, S. R. Shenoy, K. O. Rasmussen, A. Saxena, and A. R. Bishop, Phys. Rev. B (communicated).

${ }^{14}$ K. A. Gschneider, Solid State Phys. 16, 275 (1964).

15 R. W. Hertzberg, Deformation and Fracture Mechanics of Engineering Materials (Wiley, New York, 1976).
16 S. Kartha, J. A. Krumhansl, J. P. Sethna, and L. K. Wickham, Phys. Rev. B 52, 803 (1995).

17 R. Vaidyanathan, M. A. M. Bourke, and D. C. Dunand, Acta Mater. 47, 3353 (1999).

Figure Captions:

Figure 1: Spatial distribution of orientation angle $\theta(\vec{r})$ (snapshot (a)) and uniaxial strain $\epsilon_{x x}(\vec{r})$ (snapshot (b)) for stress $\sigma=2.35 \mathrm{GPa}$.

Figure 2: Average uniaxial strain $\left\langle\epsilon_{x x}\right\rangle$ for the linear elastic case as a function of the load $\sigma$. The curves correspond to a polycrystal (o) and single crystals with $\theta_{0}=0^{\circ}(\times), \theta_{0}=$ $11.25^{\circ}(+), \theta_{0}=22.5^{\circ}(*), \theta_{0}=33.75^{\circ}(\square)$ and $\theta_{0}=45^{\circ}$ $(\diamond)$.

Figure 3: Spatial distribution of the deviatoric strain in a global frame, $\epsilon_{2}(\vec{r})$, (snapshots (a),(c),(e) and (g)) and orientation angle $\theta(\vec{r})$ (snapshots (b),(d),(f) and (h)). The corresponding stress levels are $\sigma=0$ ((a) and (b)), $\sigma=2.69 \mathrm{GPa}$ ((c) and (d)), $\sigma=15 \mathrm{GPa}((\mathrm{e})$ and (f)) and $\sigma=0$ (after unloading) ((g) and (h)).

Figure 4: Average uniaxial strain $\left\langle\epsilon_{x x}\right\rangle$ for the martensite as a function of the load $\sigma$. The curves correspond to a polycrystal (o) and single crystals with $\theta_{0}=0^{\circ}(\times), \theta_{0}=7.5^{\circ}(+)$, $\theta_{0}=15^{\circ}(*), \theta_{0}=22.5^{\circ}(\square)$ and $\theta_{0}=30^{\circ}(\diamond)$. 
(a)

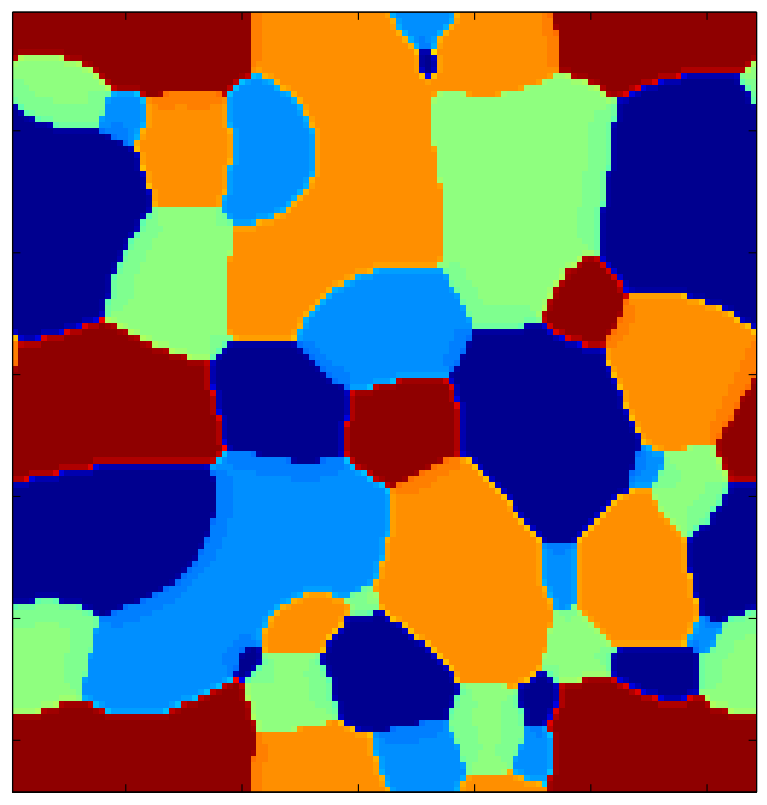

(b)

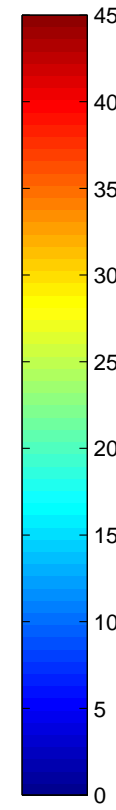

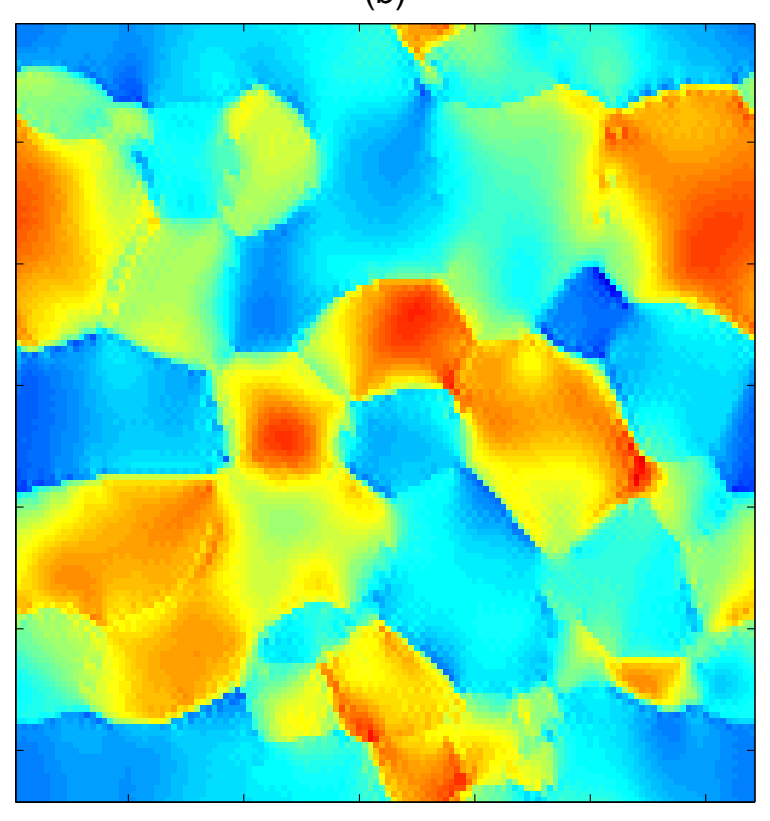

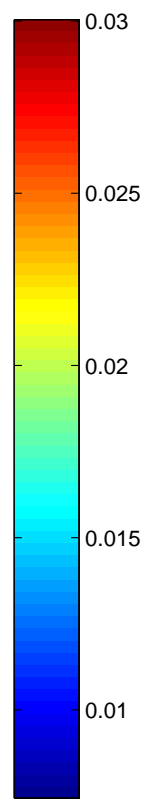




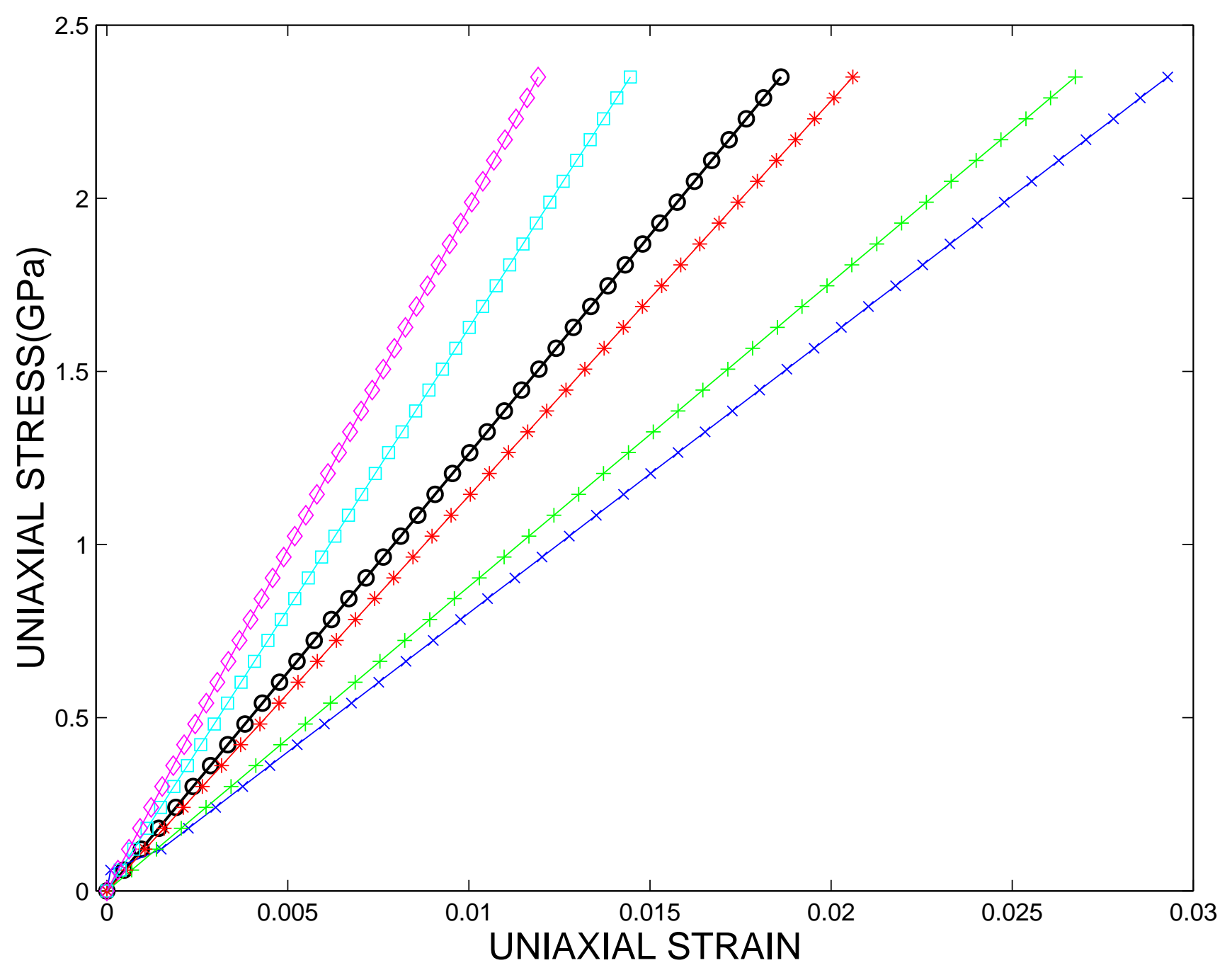



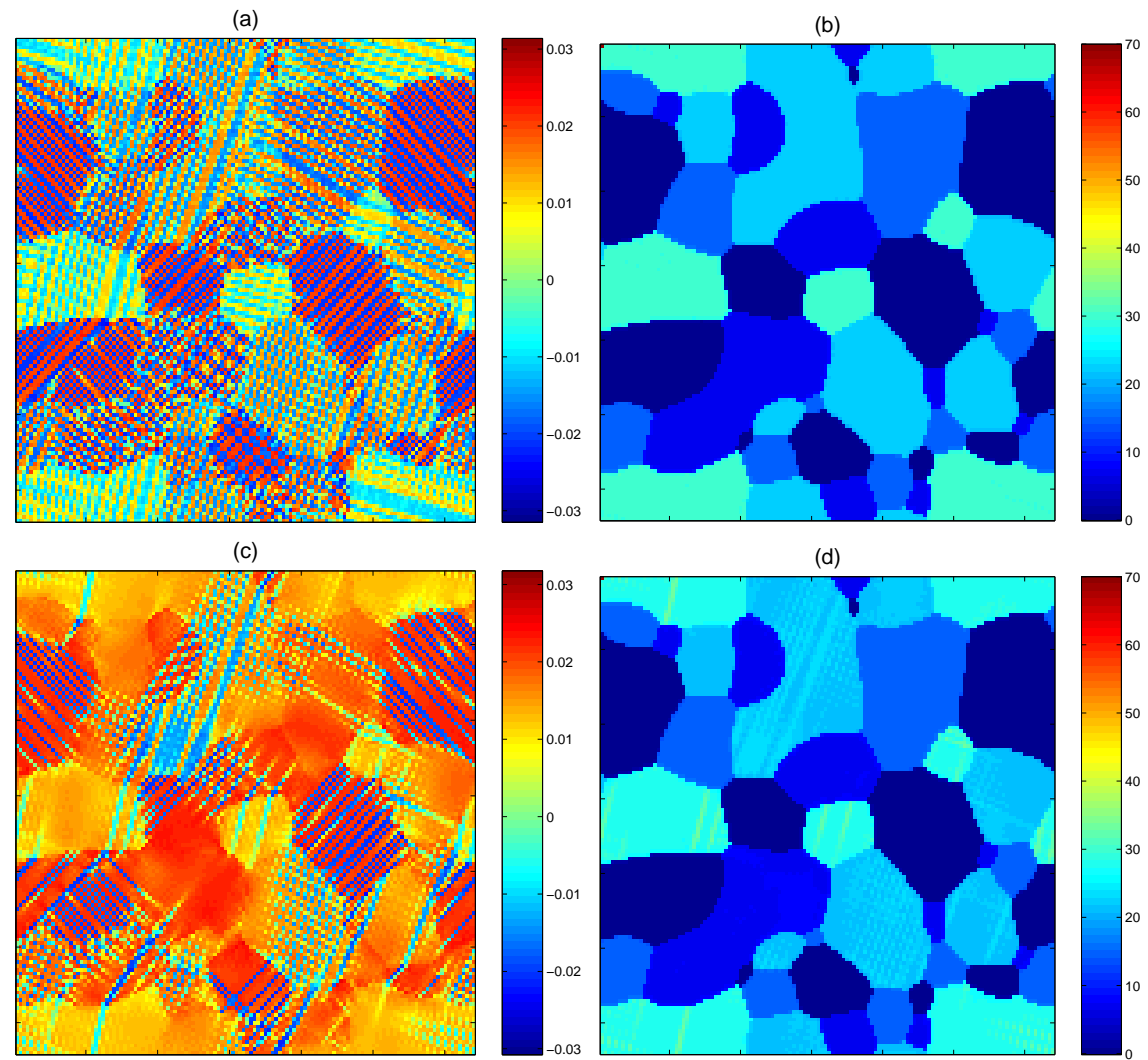

(e)
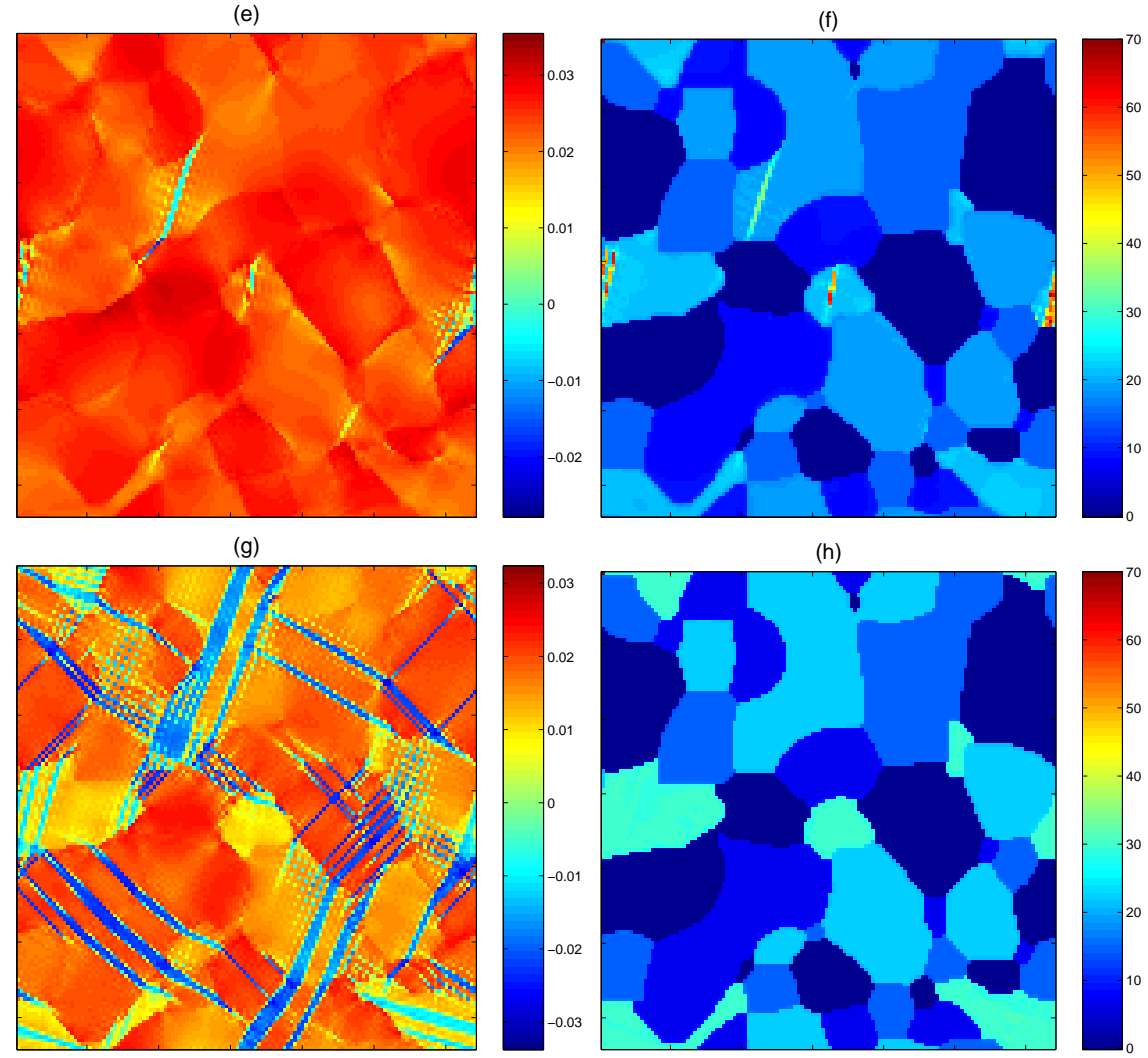


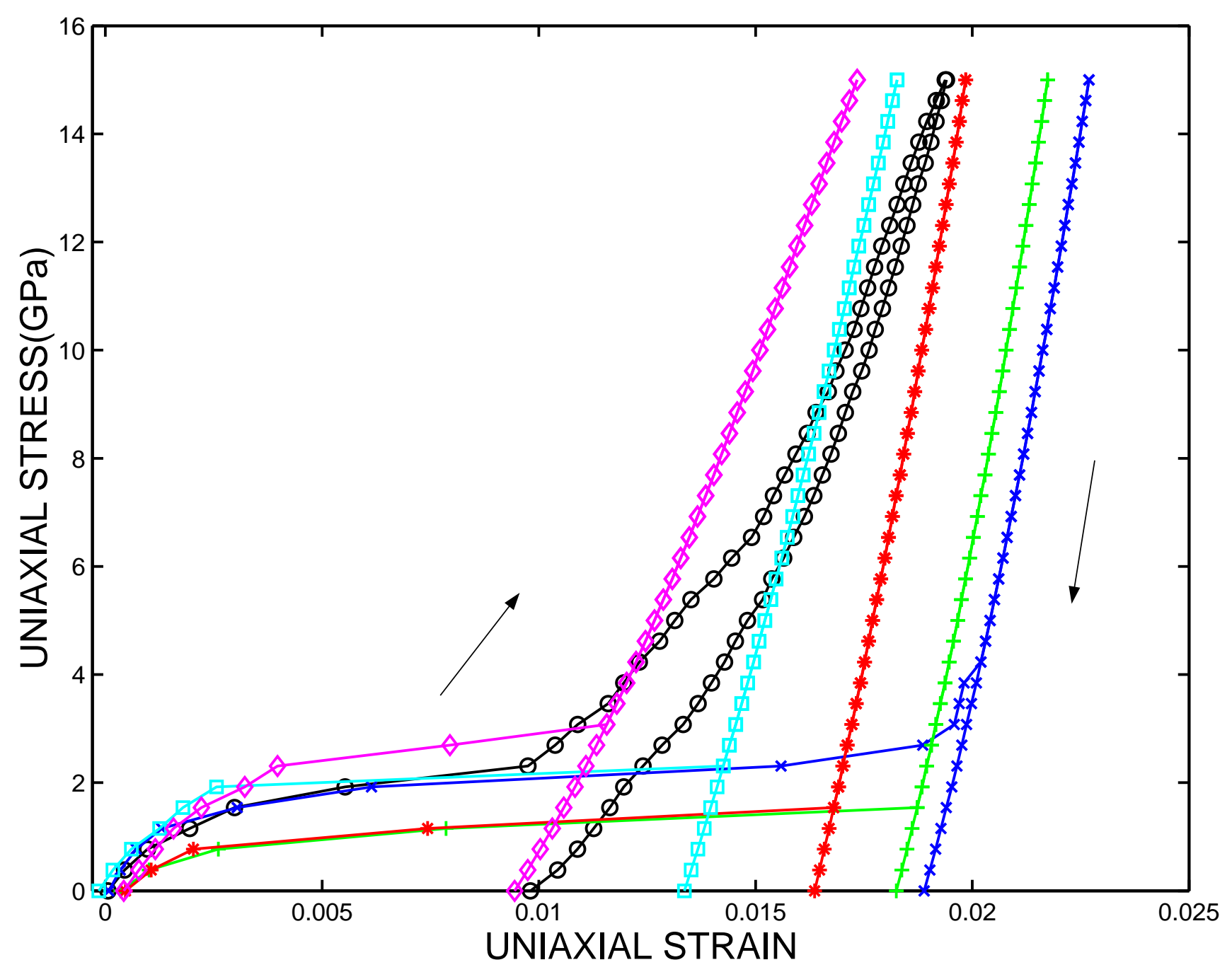

